\title{
Distractor-Induced Blindness: A Special Case of Contingent Attentional Capture?
}

\author{
Gesche N. Winther and Michael Niedeggen \\ Department of Education and Psychology, Freie Universität Berlin, Germany
}

ABSTRACT

\begin{abstract}
The detection of a salient visual target embedded in a rapid serial visual presentation (RSVP) can be severely affected if target-like distractors are presented previously. This phenomenon, known as distractor-induced blindness (DIB), shares the prerequisites of contingent attentional capture (Folk, Remington, \& Johnston, 1992). In both, target processing is transiently impaired by the presentation of distractors defined by similar features. In the present study, we investigated whether the speeded response to a target in the DIB paradigm can be described in terms of a contingent attentional capture process. In the first experiments, multiple distractors were embedded in the RSVP stream. Distractors either shared the target's visual features (Experiment $1 \mathrm{~A}$ ) or differed from them (Experiment 1B). Congruent with hypotheses drawn from contingent attentional capture theory, response times (RTs) were exclusively impaired in conditions with target-like distractors. However, RTs were not impaired if only one single target-like distractor was presented (Experiment 2). If attentional capture directly contributed to DIB, the single distractor should be sufficient to impair target processing. In conclusion, DIB is not due to contingent attentional capture, but may rely on a central suppression process triggered by multiple distractors.
\end{abstract}

\section{KEYWORDS \\ visual attention, contingent attentional capture, distractor-induced blindness}

\section{INTRODUCTION}

The never-ending stream of visual information in every-day environments on the one hand, and our limited capacity to fully process information on the other hand force us to prioritize incoming information. Most theories of visual attention assume that, apart from bottom-up mechanisms, it is the top-down information that determines whether or not a stimulus is selected for further processing (Bundesen, 1990; Desimone \& Duncan, 1995; Treisman, 1988; Wolfe, 1998). Targetdefining visual features, such as colour, form, or orientation, are thought to be specified in an attentional set, or target template, that is controlled by a network of prefrontal and parietal brain areas (Gazzaley \& Nobre, 2012). Search is guided by the attentional set, which facilitates in-depth processing of items that are similar to the target (Raymond, Shapiro, \& Arnell, 1995). To further improve the signal-to-noise ratio-enabling a more efficient target processing (Bundesen, 1990)_irrelevant stimuli are suppressed (Desimone \& Duncan, 1995). Recent evidence suggests that in case of predefined distractor features this may be achieved through a template for rejection, or a negative attentional set (Arita, Carlisle, \& Woodman, 2012; Müller, Humphreys, \& Donnelly, 1994; Woodman \& Luck, 2007; D. Zhang, Zhou, \& Martens, 2009). A negative attentional set leads to the inhibition of stimuli with distractor-like

Corresponding author: Gesche Winther, Freie Universität Berlin, Department of Education and Psychology, Raum JK 27/ 206, Habelschwerdter Allee 45, 14195 Berlin, Germany. Phone: +49 30 83875639. Mail: gesche.winther@fuberlin.de 
features (see Olivers \& Watson, 2006, for an example in rapid serial visual processing, RSVP).

However, an attentional set may not always be fine-tuned to the stimuli at hand. For example, a distractor that possesses target properties under certain circumstances may accidently be enhanced. The latter phenomenon has been termed contingent attentional capture, first described by Folk, Remington, and Johnston (1992), and observed in many different paradigms since then (e.g., Dalton \& Lavie, 2006; Ruz \& Lupiáñez, 2002). Contingent attentional capture relies on spatial shifts of the attentional focus evoked by single distractors (Folk et al., 1992; Posner, 1980). According to the contingent involuntary orienting hypothesis (Folk et al., 1992), distractors trigger an involuntary orienting response: If irrelevant stimuli share the properties of the target, these stimuli capture attention and, if presented away from the targets or if competing with target processing for other reasons, subsequently increase response times (RTs) to targets. Recent evidence suggests that the specificity of attentional control settings varies in response to different task demands, depending on what search strategy is most efficient (Atchley, Kramer, \& Hillstrom, 2000; Chua, 2015; Folk \& Remington, 1998; Moore \& Weissman, 2010). The attentional system can be set for a particular colour or a specific set of colours (e.g., Folk \& Remington, 1998; Moore \& Weissman, 2010), stimulus offset (vs. onset; Atchley et al., 2000), translational motion (vs. rotational motion; Folk, Hackman, \& Brady, 2002, as cited by Folk, Leber, \& Egeth, 2008), and rotational motion (vs. onset; Chua, 2015).

The top-down modulation assumed is manifested most prominently in spatial cueing paradigms (Ruz \& Lupiáñez, 2002). However, it has been demonstrated that a spatial shift of attention is not necessary, as the time taken to process the distractor is sufficient to impair performance: Findings regarding the contingency of attentional capture have been extended to the temporal domain, too (Dalton \& Lavie, 2006; Folk et al., 2008; Ghorashi, Zuvic, Visser, \& Di Lollo, 2003; Maki \& Mebane, 2006; Visser, Bischof, \& Di Lollo, 2004). Distractor stimuli that are presented prior to the target in an RSVP stream lead to increased RTs or even prevent the target from being detected, although no response to the distractor stimulus is required. As in the spatial domain, this phenomenon is present when distractor and target share the same visual features (Folk et al., 2008, 1992; Folk, Leber, \& Egeth, 2002).

Another mechanism induced by a (negative) attentional set has been assumed in distractor-induced blindness (DIB; Hesselmann, Niedeggen, Sahraie, \& Milders, 2006; Michael, Hesselmann, Kiefer, \& Niedeggen, 2011; Michael, Kiefer, \& Niedeggen, 2012; Niedeggen, Hesselmann, Sahraie, Milders, \& Blakemore, 2004; Sahraie, Milders, \& Niedeggen, 2001). While in contingent attentional capture the performance deficit is thought to be due to involuntary processing of a distractor preceding the target (an overshooting target template), DIB has been proposed to be based on cumulative inhibitory processes evoked by multiple distractors (an overshooting distractor template). In the DIB task (see Figure 1), two RSVPs are simultaneously presented. In the local RSVP stream, a fixation point changes its colour at a rate of $10 \mathrm{~Hz}$, while in the global stream a set of tilted bars moves randomly on the screen. Participants are instructed to attend to the local stream until its colour transiently changes to red (cue) and then instantaneously direct attention to the global stream, where in some trials all bars change their orientation (target). Participants are instructed to report the detection of the target. Occasionally, changes in orientation of the bars occur before the onset of the cue. They are defined as distractors, since they share the visual characteristics of the target and participants are instructed to ignore them.
A

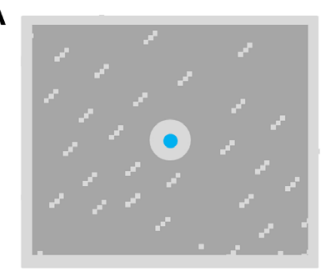

B

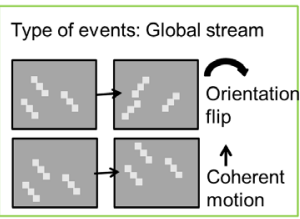

C

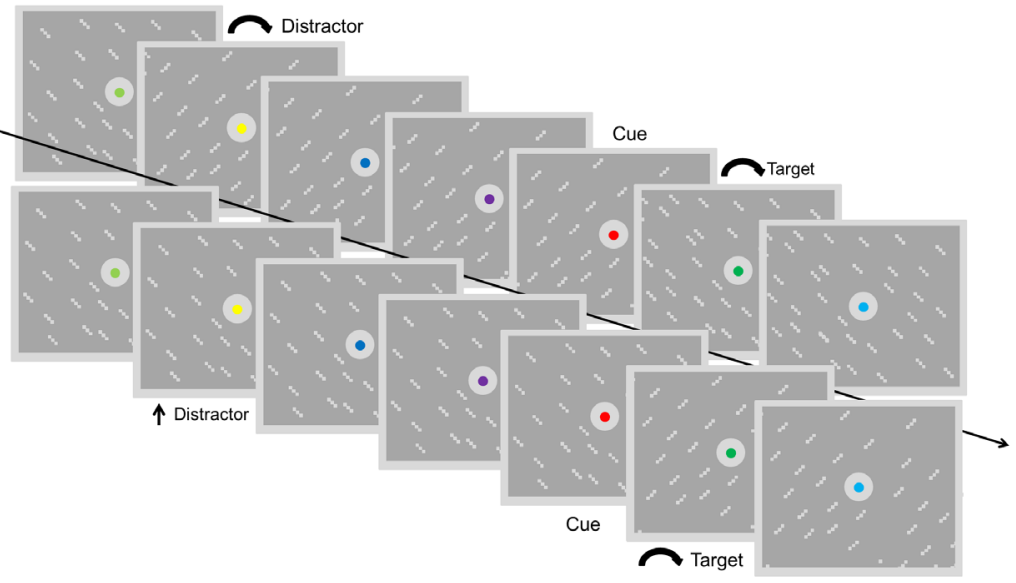

FIGURE 1.

Stimulus Configuration. A: Schematic display of global and local stimuli as presented on the screen. B: Example of the global stream event. Flip of orientation from rightwards to leftwards (above, Experiment 1A); coherent motion of the bars (below, Experiment 1B). C: Stimulus sequence, where bars in the periphery are following a random walk algorithm while the fixation circle changes its colour at a rate of $10 \mathrm{~Hz}$. Distractor events are defined as flips of orientation (above, Experiment 1A) or as coherent motion of all bars (below, Experiment 1B) in the global stream preceding the cue. The fixation points'transient colour change to red serves as the cue and the simultaneous/subsequent flip of orientation in the global stream is defined as the target. 
It has been demonstrated repeatedly that the detection of a target stimulus in the DIB paradigm is substantially impaired when it is presented in close temporal proximity to a cue and preceded by target-like distractors (Hesselmann et al., 2006; Michael et al., 2011; Sahraie et al., 2001). We assume that this effect is mainly caused by a sluggish inhibition process in response to the distractors that builds up with an increasing number of distractors and-due to its sluggish natureerroneously prevents the target from being detected (e.g., Niedeggen et al., 2004).

It has already been stated that DIB shares some properties with contingent attentional capture (Niedeggen, Michael, \& Hesselmann, 2012). In both paradigms, top-down control mechanisms are involved, since distracting stimuli are only effective when they share visual characteristics with the upcoming target. Moreover, distractors are clearly recognizable as such and are to be ignored (see, e.g., Theeuwes, Atchley, \& Kramer, 2000, for the impact of saliency and the degree of similarity). Therefore, it is likely that attentional capture-in the temporal context defined as the involuntary processing of a distractor and a subsequent impairment of target processing-may contribute to the distractor-induced blindness effect.

The present study is designed to test this hypothesis. On account of this, we evaluated RTs in addition to hit rates as a dependent measure. Studies on contingent attentional capture, especially when based on a binary response, often rely on RT measurement as the impairment by and recovery from distractor processing follows a gradual timeline (Folk et al., 2008; Ghorashi et al., 2003; but see also Pashler, 1994). In contrast to the detection rate, RTs provide a more sensitive measure of performance as they can be affected even if no difference between conditions can be found in accuracy (e.g., if hit rates are at ceiling; Prinzmetal, McCool, \& Park, 2005).

\section{EXPERIMENTS 1A AND 1B}

In Experiment 1A, the standard DIB task (Michael et al., 2011; Sahraie et al., 2001) was used: Subjects were instructed to detect a flip in orientation of the bars that appeared simultaneously with or following the onset of a colour cue (see Figure 1). The onset of the cue was either preceded by multiple distractor episodes (4-8) or by a distractor-free epoch. To allow for a direct comparison between the current speeded response design and previous DIB experiments (e.g., Hesselmann et al., 2006; Sahraie et al., 2001), the cue-target stimulus onset asynchronies (SOAs) were varied systematically.

If contingent attentional capture takes place in the current DIB design, the presentation of target-like distractors (Experiment 1A) should lead to increased RTs and reduced detection rates when presented in temporal proximity to the cue-target complex. The effect should be clearest when cue and target are presented simultaneously (SOA-0 condition). In this condition-due to the relatively short distractortarget SOA-a direct effect of distractor processing on target processing can be expected. An increasing cue-target SOA is accompanied by the increased probability that distractor processing is completed by the time of target presentation. At that point, an effect on target process- ing would only be the-potentially multiplicative-consequence of the effect the distractor had on cue processing. Furthermore, the SOA-0 condition is not contaminated by possible alternative search strategies (such as the use of the temporal distance between orientation flips as an index of target presence).

The drop in accuracy in response to distractors would replicate our earlier findings on DIB (Hesselmann et al., 2006; Michael et al., 2011; Niedeggen et al., 2012; Sahraie et al., 2001), while the additional assessment of RT data would provide a validation for the finding. Experiment $1 \mathrm{~B}$ was designed to evaluate the contingency of the visual feature of distractor and target in the speeded response task (for detection rate, see Michael et al., 2011). Distractor events consisted of a coherent motion of the bars instead of orientation flips. In contrast to Experiment 1A, distractors in Experiment 1B did not share the target's features. In a previous experiment (Michael et al., 2011) distractor features that differed from the target feature did not affect the hit rate. However, the effect on the speeded response remained to be explored. According to a contingent attentional capture account, the incongruent distractors are expected to impair neither the accuracy nor the RTs. They are assumed not to engage attentional resources and, therefore, not to affect processing of the cue-target complex.

Note that stimuli in Experiment 1B are moving randomly throughout the whole trial and do not include motion onsets (a prime suspect of bottom-up attentional capture, see Abrams \& Christ, 2003; Chua, 2015).

\section{Subjects}

Thirty-two healthy volunteers with normal or corrected-to-normal vision and normal colour vision participated in the study. Sixteen participated in Experiment 1A (12 females, aged between 20 and 32 years, $\left.M_{\text {age }}=24.31, S D=3.42\right)$ and 16 participated in Experiment $1 \mathrm{~B}$ (12 females, aged between 18 and 34 years, $M_{\text {age }}=25.63, S D=4.22$ ). All subjects gave written consent and received course credit for their participation. The study was approved by the ethics committee of the Freie Universität Berlin.

\section{Apparatus and Arrangement}

The study took place at the EEG lab of the Freie Universität Berlin. Colour vision was assessed by colour panels (Velhagen \& Broschmann, 2003). The experiment was carried out in a noise-shielded chamber with dimmed lights. The subjects were seated in front of a $21 \mathrm{in.} \mathrm{CRT}$ display (refresh rate of $100 \mathrm{~Hz}$ ) in a viewing distance of approximately $57 \mathrm{~cm}$. Stimuli were presented on the screen within a dark grey square (the square background, $25^{\circ} \times 25^{\circ}$, average luminance of $19.2 \mathrm{~cd} / \mathrm{m}^{2}$ ) that was surrounded by a light grey background (average luminance of $\left.31 \mathrm{~cd} / \mathrm{m}^{2}\right)$.

In a training session containing 40 trials, participants first learned to distinguish between target versus no-target conditions in RSVP sequences without distractors. In the second part of the training session, trials containing distractors were presented. 


\section{Stimuli, Procedure, and Design: Experiment 1A}

The main experiment consisted of 431 trials. The participant started each trial by a button press. At the beginning of each trial, 150 small bars defined by three dots each $\left(0.18^{\circ}\right.$ in diameter, average luminance of $146 \mathrm{~cd} / \mathrm{m}^{2}$ ) appeared at random locations on the square background. The bars were continuously moving following a random walk algorithm. All of them were tilted either toward the left or the right side and oriented in the same direction.

A light grey circular field $\left(3.5^{\circ}\right.$ in diameter, average luminance of $31 \mathrm{~cd} / \mathrm{m}^{2}$ ) was displayed in the centre of the square background. In the centre of this field, a coloured circle $\left(0.5^{\circ}\right.$ in diameter, average luminance of $25 \mathrm{~cd} / \mathrm{m}^{2}$ ) was presented. The circle changed its colour at a rate of $10 \mathrm{~Hz}$. During each trial of the task, observers were required to maintain fixation on it.

The participants were instructed to attend to the coloured circle (the local stream) until its colour changed to red for $100 \mathrm{~ms}$. This transient colour change served as a cue and occurred only once per trial. When detecting the cue, participants were required to decide whether the moving bars in the periphery (global stream) flipped their orientation coherently. This event was labelled as the "target". The target appeared simultaneously with the cue onset (SOA 0, 75 trials), or with an SOA of $100 \mathrm{~ms}$ (SOA 100, 66 trials), $200 \mathrm{~ms}$ (SOA 200, 60 trials), or $500 \mathrm{~ms}$ (SOA 500, 60 trials). In 90 trials, no target was presented at all. The SOA-500 condition was added to provide a baseline, with performance in both distractor conditions at ceiling.

When detecting a target, participants had to press a button on a button box (CT-3, Cambridge Research Systems) as quickly as possible. The trial continued for 1,300 ms after the appearance of the target. In trials without targets, no response was required. After cue onset, the trial continued for approximately 1,500 ms ( $\pm 200 \mathrm{~ms})$. Then, the colour changes of the fixation as well as the movement of the bars stopped, also indicating that a response was not registered any longer. The total duration of each trial was variable, depending on the duration of the pre-cue epoch.

The statistical analysis was focused on the trials in which the cue appeared at a random point in time within the interval of 2,200 to 3,000 ms after trial onset (351 out of 431 trials). In 186 trials (SOA 0, 45 trials; SOA 1, 36 trials; SOA 2, 30 trials; SOA 5, 30 trials; no target, 45 trials), flips of orientation (distractors) occurred before the cue was displayed. Participants were instructed to ignore these events. The number of distractors presented in each trial was four, six, or eight (62 trials each, 15 of which without target). The varying number of distractors was implemented to avoid temporal expectation regarding the onset of the cue. Distractors did not appear sooner than $300 \mathrm{~ms}$ after trial onset and were separated by time intervals of at least $100 \mathrm{~ms}$. The final distractor in each trial appeared at 500-800 ms before cue onset, resulting in a distractor-target SOA never exceeding $800 \mathrm{~ms}$ at (cue-target) SOA 0. Based on results of previous DIB studies (e.g., Hesselmann et al., 2006), we added more trials with distractors to the SOA-0 condition, which is characterized by a considerable rate of misses for the target. The addition of trials in this condition, therefore, intended to counterbalance the subjective probability of the different cue-target SOA conditions. DIB has been shown to be robust against changes of the proportion of trials per condition (see, e.g., Niedeggen, Busch, \& Winther, 2015).

In the remaining trials ( 80 out of 431 ), the cue appeared early (1,000-1,800 ms after trial onset). These catch trials were included to maintain the participants' attention on the local stream at the beginning of the trial and were not subject to further analysis.

The proportion of different SOA and distractor conditions in catch trials was equivalent to that in experimental trials. In catch trials, distractor conditions included fewer distractors (between one to five). The number depended on the cue onset, considering the restraints in distractor presentation that are mentioned above. After target presentation, no further orientation flip occurred.

\section{Stimuli, Procedure and Design: Experiment 1B}

In contrast to Experiment 1A, the transient events in the pre-cue epoch did not show the defining visual feature of the target (orientation flip of the elements) but were defined by a coherent motion of all bars for $100 \mathrm{~ms}$. As stated in more detail in our previous publications (Michael et al., 2011, 2012), the salience of the coherent motion is comparable to the salience of the flip of orientation and can be detected easily.

To maintain the participant's attention on the local stream, we included 41 trials without a cue (17 of which without distractors; 11 of which without targets). This was necessary because the target event differed from the distractor events. If the cue had reliably been presented on every trial, participants could have easily solved the task by waiting for the single flip of orientation in the global stream without paying attention to the local stream at all. To compensate for the additional control trials, the number of trials per experimental condition was slightly reduced. Therefore, the total number of trials (i.e., the duration of the experiment) did not differ between Experiments 1A and 1B. Sixty-six trials with an SOA of 0 ms, 57 trials with an SOA of $100 \mathrm{~ms}$, and 54 trials with an SOA of $200 \mathrm{~ms}$ and $500 \mathrm{~ms}$, respectively, were presented. (Twenty-seven trials per SOA condition were without distractors.) Seventy-nine trials did not contain a target (40 of which without distractors). Again, only trials with a cue at 2,200 to 3,000 ms were analysed ( 310 out of 431 ). The proportion of trials with zero versus four, six, and eight distractors, respectively, remained the same as in Experiment 1A (62 trials per distractor condition, 15 of which without a target and eight of which without a cue).

In both experiments, the random order of all conditions was generated offline prior to the experiment and was different for each participant. The total duration of the experiment was approximately $1 \mathrm{hr}$.

\section{Data Analysis and Results: Experiments $1 \mathrm{~A}$ and $1 \mathrm{~B}$}

In both experiments, the three distractor conditions (four vs. six vs. eight distractors within a trial) were collapsed in each analysis in order to increase the number of trials per condition. Experimental effects were analysed using a repeated-measures analysis of variance (ANOVA), including the within-subject factors of Distractors (2) and SOA (4), 
and the between-subjects factor of Feature Similarity or Experiments (2). In case of a significant interaction, Bonferroni-corrected pairwise comparisons were performed.

\section{TARGET DETECTION}

In $2.08 \%$ of the trials $\left(S D=6.08 \% ; M_{\text {ExplA }}=2.17 \%, S D=6.74 \%\right.$; $M_{\text {Exp1B }}=2.00 \%, S D=5.42 \%$ ), the button was pressed too early-that is, prior to target appearance. These trials were generally excluded from the calculation of accuracy.

In trials without targets, the false alarm rate was $10.33 \%$ on average $(S D=12.01 \%)$, indicating a rather conservative response criterion of the participants. A repeated-measures ANOVA revealed no significant distractor effect in false alarm rates, but a significant interaction with the between-subjects factor of Feature Similarity, $F(1,30)=4.85, p$ $<.04, \eta_{\mathrm{p}}{ }^{2}=.14$. Post-hoc tests showed that the trials with distractors led to a significantly higher false alarm rate in Experiment $1 \mathrm{~A}, t(15)=$ $-2.70, p<.02$, but not in Experiment $1 \mathrm{~B}(p>.05)$.

Following Figure 2A, the expected effect of DIB on target detectability can also be observed in a speeded response setup: The hit rate was decreased by the presence of distractors, and this effect recovered as a function of cue-target SOA. However, this was only the case if distractors shared the target features (Experiment 1A); if distractors differed from targets (Experiment 1B), hit rates appeared to be generally at ceiling. The ANOVA confirms this observation: Although main effects of distractors, $F(1,30)=24.75, p<.01, \eta_{\mathrm{p}}{ }^{2}=.45$, and SOA, $F(3,90)=20.99, p<.01, \eta_{\mathrm{p}}{ }^{2}=.41$, GG $\varepsilon=.45$, were highly significant, they critically depended on Feature Similarity (Distractor $\times$ Feature Similarity: $F[1,30]=26.35, p<.01, \eta_{\mathrm{p}}{ }^{2}=.47$; SOA $\times$ Feature Similarity: $\left.F[3,90]=16.56, p<.01, \eta_{\mathrm{p}}{ }^{2}=.36\right)$. The same applied to the Distractor $\times$ SOA interaction, $F(3,90)=19.85, p<.01, \eta_{\mathrm{p}}{ }^{2}=.40$, which was also modulated by Feature Similarity (Distractor $\times \mathrm{SOA} \times$ Feature Similarity: $F[3,90]=21.40, p<.01, \eta_{\mathrm{p}}^{2}=.42$ ). Post-hoc tests confirmed that significant effects of distractor presentation were only obtained in Experiment $1 \mathrm{~A}$ and that these effects were more expressed at shorter SOAs (SOA 0: $t[15]=-6.31$; SOA 100: $t[15]=-4.35$; SOA 200: $t[15]$ $=-3.69 ; p<.05$ for each one). In the critical SOA-0 condition, targets were detected in $59.54 \%$ of trials, which is more than $30 \%$ below that of trials without distractors. A corresponding effect was not observed for the corresponding post-hoc tests in Experiment 1B ( $p>.05$ for all
SOA conditions). Please note that the experimental procedure did not require a forced-choice discrimination concerning the expression of a target feature, but its mere detection. In an earlier study (Niedeggen, Sahraie, Hesselmann, Milders, \& Blakemore, 2002), we demonstrated that discrimination is at chance level if the target is not detected. The overall proportion of guesses is-with reference to the relatively low false alarm rate-thought to be minor.

\section{REACTION TIME}

Participants' correct RTs (see Figure 2B) were analysed. In order to reduce the influence of outliers and increase the statistical power, all RTs that were further from the participants' mean RT than 2 SDs were not included into the analysis (Ratcliff, 1993; van Zandt, 2002).

Following Figure 2B, the mean RT was increased if distractors were presented, and this effect appears to be reduced with increasing cuetarget SOA. Again, there is a distinct distractor effect at SOA 0. In line with the hit rates, this effect appears to be present only in Experiment $1 \mathrm{~A}$ but not in Experiment 1B. This observation was confirmed by the ANOVA indicating main effects of distractor, $F(1,30)=22.95, p<.01$, $\eta_{\mathrm{p}}{ }^{2}=.43$, and SOA, $F(3,90)=152.79, p<.01, \eta_{\mathrm{p}}{ }^{2}=.84$, GG $\varepsilon=.50$, as well as an interaction of both factors, $F(3,90)=4.57, p<.01, \eta_{p}{ }^{2}=$ .13. Most importantly, the main effects as well as the interaction were significantly modulated by the factor of Feature Similarity (Distractor $\times$ Feature Similarity: $F[1,30]=14.01, p<.01, \eta_{\mathrm{p}}{ }^{2}=.32$; SOA $\times$ Feature Similarity: $F[3,90]=4.34, p<.01$; Distractor $\times$ SOA $\times$ Feature Similarity: $\left.F[3,90]=2.85, p=.04, \eta_{\mathrm{p}}{ }^{2}=.09\right)$. The post-hoc comparison confirmed that significant differences between distractor conditions were restricted to Experiment 1A. At the critical SOA-0 condition and at SOA $100, t(15)=3.29$ and $t(15)=5.04$, respectively, $p<.01$ for both, but not at SOA 200 ( $p>.05)$, RT was significantly reduced by the presence of distractors. Even though the difference between mean RTs at SOA 500 was significant, too, $t(15)=3.26, p<.01$, it was smaller than at SOA 0 and SOA 100. In Experiment 1B, distractor conditions did not differ significantly from no-distractor conditions at any SOA $(p>.05)$.

\section{Discussion: Experiments $1 \mathrm{~A}$ and $1 \mathrm{~B}$}

Our data replicated the results of previous studies on DIB (e.g., Hesselmann et al., 2006; Niedeggen et al., 2004; Sahraie et. al., 2001). Participants missed significantly more targets at SOA 0 when target-
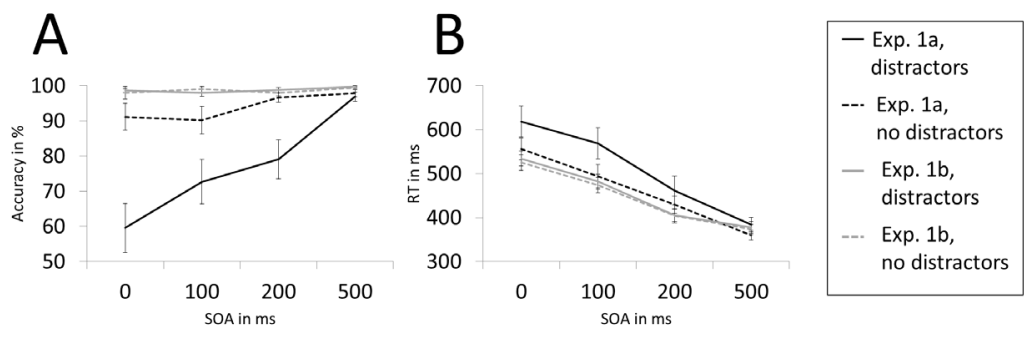

FIGURE 2.

Plot of accuracy data (A) and mean reaction time (RT) (B) of conditions with distractors (solid line) as compared to conditions without distractors (dashed line) in Experiment 1A (black ink) and Experiment 1B (grey ink). It shows that differences between conditions are most pronounced at shorter SOAs. Error bars denote the standard error of the mean. 
like distractors had been presented previously. First, this confirms that the effect of DIB can be reliably evoked in a speeded response setting. Second, we were able to replicate our earlier findings on the feature specificity of the effect (Michael et al., 2011, 2012) that suggest a topdown controlled inhibition of a task-set.

The finding is also in line with a contingent attentional capture explanation: If the distractors match the target template (Experiment 1A), they capture attention and, therefore, occupy processing resources. Since the last distractor is presented in close temporal proximity to the cue-target complex, its processing impairs the processing of cue and/or target. As predicted by the contingent involuntary orienting hypothesis (Folk et al., 1992), distractors that do not match the target template (Experiment 1B) do not have such an effect.

RTs to correctly detected targets are affected by the presence of distractors in a comparable manner. Distractors lead to an increase at the overall RT level, especially at early SOAs. The RT effect shares the characteristics of the aforementioned effect on the hit rate: It critically depends on the similarity of the visual features of distractors and the upcoming target. The effect is not elicited by a visual transient that is irrelevant for target processing (Experiment 1B). However, data also reveal differences between the behavioural measures: The RT effect appears to be longer lasting and is even present at SOA 500. In contrast, the effect on hit rate appears to be more short-lived. Despite these effects, the RT results are in line with the DIB account, indicating that distractor presentation does not only affect the detection performance but also the motor response to a detected target. Following our model, the cumulative inhibition is not immediately released in case of successful target detection. The fact that performance is reliably affected by distractor presentation when cue and target are presented simultaneously, or with a short delay, however, can also be explained in terms of a contingent attentional capture account (Folk et al., 1992) : Distractors sharing the target's features transiently capture the participants' attention, thereby impairing performance and delaying the preparation of the motor response. Even though this process is triggered involuntarily, the effect is not entirely bottom-up driven but contingent upon the current task set (here: to detect a flip of orientation).

\section{EXPERIMENT 2}

In Experiment 1, we presented multiple distractors. In the second experiment, we abolish this defining element and present a single distractor instead. An important prediction derived from earlier findings on contingent attentional capture is that one single distractor presented in temporal proximity to the target should be sufficient to elicit the effect. This has been demonstrated in standard tasks in the spatial domain (viz., spatial cueing, e.g., Folk et al., 1992; Posner, 1980, and visual search, see Wolfe, 1998, for a review) as well as for the temporal domain (Folk et al., 2008; Maki \& Mebane, 2006; Raymond, Shapiro, \& Arnell, 1992).

Previous studies suggested that the presentation of multiple distractors may reduce a potential capture effect of the last distractor. This reduction may occur due to adaptation/training processes (for fre- quency effects between trials see Cosman \& Vecera, 2010; Horstmann \& Ansorge, 2006; Kelley \& Yantis, 2009; Neo \& Chua, 2006; for topdown adjustment within trials see Irons \& Remington, 2013) or due to attentional engagement/capture by the preceding distractor (Folk, Ester, \& Troemel, 2009; Moore \& Weissman, 2011; but see Zivony \& Lamy, 2014). Following these findings, the RT results in Experiment $1 \mathrm{~A}$ can be assumed to underestimate the distractor effect. If a comparable mechanism affects target processing in contingent attentional capture and DIB, then the distractor effect in Experiment 1A does not rely on the repeated distractor presentation but on the presentation of the final distractor in temporal proximity to the cue. Its processing may hinder target processing after all. One single distractor at the same position as this last distractor should therefore be sufficient to induce an increase in RTs and a decrease in hit rates in the DIB task. As the distractor shares the targets' features, it will involuntarily draw attention, thus affecting the processing of the target.

Experiment 2 tested for these predictions. A single distractor was placed in the same temporal relation to the cue as had been the last distractor in Experiment 1. According to the results on contingent attentional capture, the RT pattern and the hit rates in Experiment 2 should be comparable to that obtained in Experiment 1A. The focus of interest is, again, the condition in which cue and target are presented simultaneously. In case of attentional capture, accuracy should be decreased and RTs should be increased at SOA 0 .

\section{Subjects}

A set of 16 healthy volunteers (14 females, between 18 and 35 years of age, $\left.M_{\text {age }}=23.19, S D=5.10\right)$ with normal or corrected-to-normal vision and normal colour vision participated in the study. None of the subjects had participated in Experiment 1. All subjects gave written consent and received course credit for their participation. The study was approved by the ethics committee of the Freie Universität Berlin.

\section{Stimuli, Procedure, and Design}

In experimental conditions, only a single distractor in temporal proximity to the cue was presented. Its position was 500-800 ms before cue onset, equivalent to the position of the last distractor in Experiment 1. The target appeared either at SOA 0, 100, 200, or 500 (56 trials per SOA condition; each consisting of 28 trials with and 28 without a distractor). To account for false alarms, in 84 trials (each consisting of 42 with and without a distractor), no target was displayed at all. As in Experiment 1, the cue appeared between 2,200 and 3,000 ms after trial onset.

In order to reduce the predictability, two control conditions were introduced. In 52 trials (10 trials per SOA condition, 12 trials without target), the distractor event was uncorrelated with the cue and appeared at a random position between $300 \mathrm{~ms}$ after trial onset and $800 \mathrm{~ms}$ before cue onset. These trials were included in order to prevent the distractor from serving as a warning stimulus (Fischer, Plessow, \& Ruge, 2013; Hackley, 2009; Hackley \& Valle-Inclán, 2003; Niemi \& Näätänen, 1981). On the one hand, those trials, even though excluded from analysis, led to a wider range of distractor-cue SOAs (which made the experiment different from Experiment $1 \mathrm{~A}$ in that respect). 
On the other hand, participants could not know whether a second distractor would be presented by the time of distractor presentation, which makes the situation similar to that of Experiment 1A. In both experiments, the temporal relation of a certain distractor to the target could only be known afterwards. That makes a differential effect on capture rather unlikely. In 27 trials ( 5 trials per SOA condition, 7 trials without target), two distractors preceded the appearance of the cue. This condition was added to reduce the predictability of the number of orientation changes (comparable to the varying number of distractors in Experiment 1). In both control conditions, the cue appeared between 2,200 and 3,000 ms after trial onset.

As in Experiment 1, catch trials with early cue onset (1,000 to 1,800 ms after trial onset) were included ( 5 trials per condition, 50 trials in total). The control trials (129 of 437) were not considered for further analysis. The random order of all 437 trials was generated offline, separately for each participant.

Because of the two additional control conditions in Experiment 2 (single random distractor and two distractors), the overall number of distractors as well as the number of trials per condition differed marginally from that of Experiments $1 \mathrm{~A}$ and $1 \mathrm{~B}$. However, this slight imbalance in the distribution of trials across conditions is unlikely to affect the behavioural effects (see above, Niedeggen et al., 2015).

\section{Data Analysis and Results}

Experimental effects were analysed using a repeated-measures ANOVA, with factors of Distractor (2) and SOA (4).

\section{TARGET DETECTION}

Trials without targets led to a false alarm rate of $12.28 \%$ on average $(S D=11.12)$. Again, trials including a distractor led to a significantly higher false alarm rate $(M=17.86, S D=12.84)$ than trials without a distractor $(M=6.70, S D=4.94), t(15)=4.10, p<.01$. In comparison to Experiment 1A, however, the false alarm rate was not significantly different. In $1.51 \%(S D=3.22 \%)$ of the trials, the button was pressed too early. Hit rates were calculated as in Experiment 1.
As shown in Figure 3A, the target detection rate exceeds $85 \%$ in all conditions. However, the presentation of a single distractor had a modest but reliable effect on the accuracy. The ANOVA revealed a significant main effect of distractor, $F(1,15)=11.24, p<.01, \eta_{\mathrm{p}}^{2}=.43$, with conditions including a distractor showing significantly lower accuracy rates than conditions without a distractor. The main effect of SOA was also significant, $F(3,45)=11.73, p<.01, \eta_{\mathrm{p}}{ }^{2}=.44, \mathrm{GG} \varepsilon=$ .49. Here, shorter SOAs led to lower hit rates. The interaction between the factors SOA and Distractor was significant, $F(3,45)=4.79, p<.01$, $\eta_{\mathrm{p}}{ }^{2}=.24$. Post-hoc tests, like in Experiment 1A, showed a reliable effect at SOA $0, t(15)=3.47, p<.01$. As depicted in Figure 3B, hit rates were approximately $10 \%$ lower in trials with distractors as compared to trials without distractors. The effect was significant at SOA 100 , too, $t(15)=$ $2.87, p<.01$. To substantiate the finding, we compared the distractor effects on hit rates in Experiments $1 \mathrm{~A}$ and 2. We focused the analysis on the SOA-0 condition, assuming that a potential capture effect is most pronounced and least contaminated here (see above). Previous analyses have shown that the effect was most distinct in this condition: As depicted in Figure 3B, in both experiments, hit rates are decreased in trials with distractors. This effect is more pronounced in Experiment 1A. Accordingly, the repeated-measures ANOVA (Experiment $\times$ Distractor) revealed significant main effects of distractor, $F(1,30)=$ 51.82, $p<.01, \eta_{\mathrm{p}}{ }^{2}=.63$, and experiment, $F(1,30)=6.89, p=.01, \eta_{\mathrm{p}}{ }^{2}=$ .19. The effect of distractor differed significantly between experiments, indicating a more pronounced distractor effect in Experiment $1 \mathrm{~A}$ as compared to Experiment 2 (interaction of Experiment $\times$ Distractor: $\left.F[1,30]=16.06, p<.01, \eta_{\mathrm{p}}^{2}=.35\right)$.

\section{REACTION TIME}

As in Experiment 1, all RTs exceeding the participants' mean RT by more than two SDs were not included in the analysis. Data are shown in Figure 3B. Comparable to Experiment 1, there was a significant main effect of SOA, $F(3,45)=66.89, p<.01, \eta_{\mathrm{p}}^{2}=.82$, GG $\varepsilon=.48$, with significantly faster RTs for longer SOAs. In contrast to Experiment $1 \mathrm{~A}$, RTs did not differ between conditions with distractors and conditions

FIGURE 3.

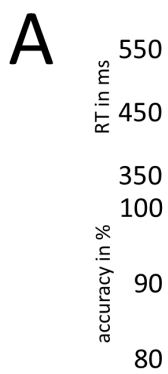

80
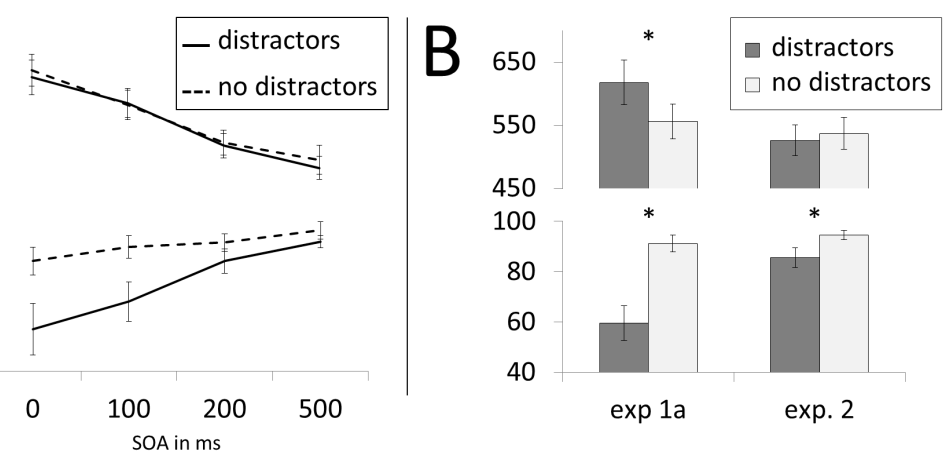

A: Plot of mean reaction time (RT) data (above) and accuracy data (below) of Experiment 2. The solid line represents mean responses to conditions with distractors whereas the dashed line represents mean responses to conditions without distractors. B: Plot of mean RT data at SOA 0 (above) and mean accuracy data at SOA 0 (below) of Experiment $1 \mathrm{~A}$ (left) and experiment 2 (right). Trials with distractors are represented by dark grey bars, trials without distractors are represented by light grey bars. Error bars denote the standard error of the mean. 
without distractors, the interaction of Distractor $\times$ SOA was not significant either $(p>.05)$. A direct comparison between trials with and without distractors at SOA 0 did not reveal a significant effect either $(p$ $\left.=.41, \eta_{\mathrm{p}}^{2}=.05\right)$.

RT effects were compared between experiments by a repeated-measures ANOVA with within-subject factors of SOA (4) and Distractor (2) and a between-subjects factor of Experiment (2). Corresponding to the analysis on hit rate, only the SOA- 0 condition was considered. While participants in Experiment 1A responded about $60 \mathrm{~ms}$ slower when distractors were presented, the difference in Experiment 2 was marginal (see Figure 3B). The ANOVA revealed a significant main effect of distractor, $F(1,30)=4.95, p=.03, \eta_{\mathrm{p}}{ }^{2}=.14$, as well as a significant interaction of Distractor $\times$ Experiment, $F(1,30)=10.21, p<.01, \eta_{\mathrm{p}}{ }^{2}=$ .25. The interaction was due to the fact that in Experiment $1 \mathrm{~A}$ distractors led to decreased RTs, whereas the distractor in Experiment 2 had no such effect.

\section{Discussion: Experiment 2}

The data of Experiment 2 were not in line with a contingent attentional capture-based model and indicate that DIB relies on a different mechanism. With respect to hit rates, the performance was affected by the presentation of a single distractor. This effect was only present at early cue-target SOAs. However, the direct comparison with Experiment 1A confirmed that the effect elicited by a single distractor was significantly reduced as compared to the effect of multiple distractors. The results are, therefore, congruent with earlier findings indicating that a cumulative suppression process is triggered by the presentation of multiple distractors (Hesselmann et al., 2006). Direct evidence for the additive effect of distractors was provided in ERP experiments (Niedeggen et al., 2012).

More importantly, RTs were not impaired by the presentation of a single distractor. In Experiment 2, neither a significant distractor effect nor a significant Distractor $\times$ SOA interaction was obtained. At SOA 0 , no significant difference was found. This pattern of results is clearly in contrast with predictions based on contingent attentional capture findings, where a single distractor is always sufficient-if not more successful (Folk et al., 2009; Irons \& Remington, 2013)—in inducing a negative effect (Folk et al., 2008, 1992).

The missing distractor effect on the RT level is even more compelling, as the final distractor in Experiment 1A was presented at the very same temporal position as the single distractor in Experiment 2. Differences can therefore not be attributed to differences in the SOA between distractor and target, which appears to be crucial for the inhibitory effect of distractors in contingent attentional capture (e.g., Ghorashi et al., 2003).

However, one has to consider that the results may also imply that the changed number of distractors in Experiment 2 entailed inherent changes of the setting, making the task itself easier. These inherent changes would be relevant for the notion of attentional capture if they affected distractor perception and processing, for example, due to decreased saliency. Previous DIB studies suggest that it is very unlikely that a single distractor in DIB is less salient, as its perception is not impaired by the cumulative suppression in response to previous distractors (Niedeggen et al., 2015, 2012). Furthermore, the sensory response to the first distractor has been shown to be more expressed as compared to the response to the succeeding distractors (Niedeggen et al., 2004, 2012), indicating that a single distractor is sufficiently represented at least at a sensory level. This corresponds to the findings regarding multiple distractors in attentional capture which suggest that a single distractor is not less but more prone to attentional capture (Cosman \& Vecera, 2010; Folk et al., 2009; Horstmann \& Ansorge, 2006; Irons \& Remington, 2013; Kelley \& Yantis, 2009; Neo \& Chua, 2006). Together with the finding that the design was sufficiently difficult to detect distractor effects at all (see accuracy at SOA 0), we assume that Experiment 2 was an appropriate test for the attentional capture hypothesis.

\section{GENERAL DISCUSSION}

\section{Summary of the Results}

In a series of three experiments, we examined whether the cumulative effect of distractors in DIB may be explained on the basis of contingent attentional capture. Since distractors were embedded in an RSVP stream and only led to a significant effect when they shared the visual characteristics with the upcoming target (Michael et al., 2011), we initially assumed that contingent attentional capture (Folk et al., 1992) may be directly responsible for DIB.

Data obtained in Experiment 1A were in line with this assumption: Presentation of multiple target-like distractors led to significantly increased RTs and reduced accuracy, and the effect was reduced with an increased temporal delay between the onset of the final distractor and the target. This effect did not occur when the distractor features differed from the target features (Experiment 1B). However, a single target-like distractor at the same temporal position as the final distractor in Experiment 1 did not have an effect on RT (Experiment 2). This implies that the DIB effect is not directly due to an involuntary processing of the final distractor presented in temporal proximity to the cue.

Furthermore, the data indicate that RT effects and response accuracy are dissociated in the classic DIB task. These findings will be discussed in the following.

\section{Impact of Contingent Attentional Capture in Distractor-induced Blindness}

Despite of the striking parallels between contingent attentional capture and DIB, our data do not provide evidence that comparable mechanisms are triggered in the DIB design. This finding leads to two further questions.

First, why is contingent attentional capture not observed in our temporal selection task relying on multiple distractor presentation? One might assume that the temporal interval between the final distractor and the target onset (>500 ms) contributes to the difference. As already pointed out, the capture effect is more expressed for shorter 
temporal intervals (Ghorashi et al., 2003; Leblanc \& Jolicour, 2005). Following this idea, the lack of an RT effect in DIB does not indicate that distractors were not processed at all. Rather, attention may already be disengaged from the distractor by the presentation of the target, thus not affecting its processing (Theeuwes et al., 2000). However, this does not suffice as an explanation: It has been demonstrated that temporal attentional capture in an RSVP setting can last $500 \mathrm{~ms}$ and more (Folk et al., 2008; Lamy, Leber, \& Egeth, 2004; Liao \& Yeh, 2013; Maki \& Mebane, 2006). This time range also applies to our experiments: In Experiment 2, the final distractor was presented 500-800 ms before target onset (the SOA- 0 condition) and did not affect the RT.

However, we have to consider that attentional capture is most pronounced when the distractor-target SOA averages $200 \mathrm{~ms}$ or less (Folk et al., 2002; Lamy et al., 2004; Leblanc \& Jolicour, 2005) and that some studies have failed to show a capture effect at distractor-target SOAs exceeding 350 ms (e.g., Leblanc \& Jolicœur, 2005; Moore \& Weissman, 2010). Even minor changes in stimulus array had a large impact on the duration of attentional capture (e.g., Leblanc \& Jolicour, 2005, as compared to Lamy et al., 2004). Leblanc and Jolicœur (2005) pointed out that the factors that influence the magnitude of attentional capture, such as stimulus duration, salience, and eccentricity, are very likely to affect its time course, too. The stimuli's feature dimension (Girelli \& Luck, 1997) could also have an impact. Only recently, a study by Liao and Yeh (2013) suggested that attentional capture by dynamic features (viz., onsets) may be less persistent than attentional capture by static features (but mind onset's special status regarding attentional capture, see Liao \& Yeh, 2013). Another important aspect is the search mode (e.g., Folk \& Remington, 1998). Conjunction search is thought to be executed differently than feature search (Wolfe, 1998) and may be less susceptible to attentional capture (Lamy \& Tsal, 1999, but see Maki \& Mebane, 2006, for "successful" contingent attentional capture in a conjunction RSVP task). Taking into account that the current task could also partially be interpreted as a conjunction search task (response to the combination of red fixation and peripheral orientation flip), this latter aspect may be crucial with regard to the missing capture effect in DIB. Moreover, the relation of target and distractor properties (see Theeuwes et al., 2000, for the influence of distractor-target-similarity on attentional dwell time) as well as comprehensive properties, such as perceptual load (Cosman \& Vecera, 2010), have to be considered, too.

Considering these restrictions, we cannot rule out that attentional capture would affect the DIB effect in case of short distractor-cue SOAs. However, we found evidence that attention is not captured by distractors at the time of target presentation in the "classic" DIB design (i.e., with distractor-cue SOAs $>500 \mathrm{~ms}$ ).

Assuming an independence of contingent attentional capture and DIB, the second question can be derived: If contingent attentional capture does not play a role in the DIB paradigm, which processes are responsible for the distractor effect in the task? Past studies related the DIB to a cumulative frontal inhibitory process (e.g., Niedeggen et al., 2004, 2012). This process is proposed to prevent an updating of the distractor representation in working memory, thus reducing the conscious access to stimuli sharing the same visual feature (Niedeggen et al., 2012). A similar model has been developed for the attentional blink (boost and bounce theory, Olivers \& Meeter, 2008). Both assume that a gating system is activated by distractors preventing an incorrect answer (Desimone \& Duncan, 1995; Engle, Conway, Tuholski, \& Shishler, 1995; Olivers \& Meeter, 2008). The present data further narrow down the characteristics of that process by showing that not only the perceptual but also the (pre)motoric level (as evaluated by means of RT in correct responses) is affected.

This, in a way, is the complement to the role of contingent attentional capture. While contingent attentional capture is triggered by an overshooting positive attentional set (focus on target properties), DIB is due to an overshooting negative attentional set (suppression of distractors, see also Olivers \& Watson, 2006; D. Zhang et al., 2009). On account of this, it appears logical that DIB is influenced by the number of distractors while no such evidence is found for attentional capture.

\section{Dissociation Between Response Time and Accuracy in Distractor- Induced Blindness}

The assumption of an inhibitory mechanism activated in DIB by repeated distractor presentations is based on accuracy data (Hesselmann et al., 2006; Niedeggen et al., 2004, 2002). In the series of experiments presented here, a speeded response procedure was applied for the first time. The new setup allowed for an extended insight into the processes involved in DIB.

Previous studies on visual attention already indicated that the underlying processes of accuracy and RT data can differ (Prinzmetal et al., 2005; van Ede, de Lange, \& Maris, 2012). In Experiment 1A, a significant distractor effect was obtained for both RT and accuracy. This is in line with the proposition that distractors lead to a conflict at the response level but also at the level of stimulus identification $(\mathrm{H}$. H. Zhang, Zhang, \& Kornblum, 1999). However, we initially assumed that RT and accuracy are affected by the same underlying processes in DIB. Following this idea, an effective inhibition can prevent the access to the target ("miss") but will also slow down the response in case of target detection. Accordingly, the release functions of RT and accuracy data should be the same. As shown in Figure 2, this was not the case. Which different processes are exactly involved in DIB would have to be the subject of future research.

\section{CONCLUSION}

According to the present results, contingent attentional capture does not appear to be directly responsible for the effect that is observed in DIB. The lack of an observable capture effect elicited by a single distractor in the DIB design might be attributed to the feature-sensitivity of the process, or to its, more transient, time effect.

However, RTs in the DIB paradigm were clearly affected by multiple distractors. Our results support the idea that different facets of the central suppression process involved (Niedeggen et al., 2012) are reflected by response accuracy and speeded motor response. 


\section{AUTHOR NOTE}

This work was supported by the German Research Foundation under Grant NI 513/8-2. The authors would like to thank Guido Hesselmann for his support in preparing the experiment. The authors declare no conflict of interest.

\section{FOOTNOTES}

${ }^{1}$ Some other explanations that could account for the present data have been ruled out by previous DIB studies; see, for example, Hesselmann et al. (2006) for a study that provides evidence against distractor-induced uncertainty of temporal cue-target order as a source of the effect.

${ }^{2}$ Note that in the current study we did not intend to make the design prone to contingent attentional capture. Rather, we intended to test for the eventual contribution of attentional capture to "classic" DIB in its original design, which is why, for example, we did not shorten the original distractor-target SOA.

\section{REFERENCES}

Abrams, R. A., \& Christ, S. E. (2003). Motion onset captures attention. Psychological Science, 14, 427-432. doi: 10.1111/14679280.01458 [WW|

Arita, J. T., Carlisle, N. B., \& Woodman, G. F. (2012). Templates for rejection: configuring attention to ignore task-irrelevant features. Journal of Experimental Psychology: Human Perception and Performance, 38, 580-590. doi: 10.1037/a0027885 (WwW

Atchley, P., Kramer, A., \& Hillstrom, A. (2000). Contingent capture for onsets and offsets: Attentional set for object transients. Journal of Experimental Psychology: Human Perception and Performance, 26, 594-606. doi: 10.1037/0096-1523.26.2.594 WWW

Bundesen, C. (1990). A theory of visual attention. Psychological review, 97, 523-547. doi: 10.1037/0033-295X.97.4.523 |www

Cambridge Research Systems Ltd, 80 Riverside, Sir Thomas Longley Road, ROCHESTER, Kent, ME2 4BH, UK. Retrieved from: http://www.crsltd.com/ www

Chua, F. (2015). Attentional capture by dynamic cues. Attention Perception, \& Psychophysics, 77, 111-127. doi: 10.3758/s13414014-0759-3 [www]

Cosman, J. D., \& Vecera, S. P. (2010). Attentional capture under high perceptual load. Psychonomic Bulletin \& Review, 17, 983986. doi: 10.3758/PBR.17.6.815 WwW

Dalton, P., \& Lavie, N. (2006). Temporal attentional capture: Effects of irrelevant singletons on rapid serial visual search. Psychonomic Bulletin \& Review, 13, 881-885. doi: 10.3758/ BF03194013 www

Desimone, R., \& Duncan, J. (1995). Neural mechanisms of selective visual attention. Annual Review of Neuroscience, 18, 193222. doi: 10.1146/annurev.ne.18.030195.001205

Engle, R. W., Conway, A. R. A., Tuholski, S. W., \& Shishler, R. J. (1995). A resource account of inhibition. Psychological Science, 6, 122125. doi: 10.1111/j.1467-9280.1995.tb00318.x
Fischer, R., Plessow, F., \& Ruge, H. (2013). Priming of visual cortex by temporal attention? The effects of temporal predictability on stimulus (-specific) processing in early visual cortical areas. Neurolmage, 66, 261-269. doi:S1053-8119(12)01095-6 WwW

Folk, C. L., Ester, E. F., \& Troemel, K. (2009). How to keep attention from straying: Get engaged! Psychonomic Bulletin \& Review, 16, 127-132. doi: 10.3758/PBR.16.1.127 [wW]

Folk, C. L., Leber, A. B., \& Egeth, H. E. (2002). Made you blink! Contingent attentional capture produces a spatial blink. Perception \& Psychophysics, 64, 741-753. doi: 10.3758/ BF03194741 |WWW|

Folk, C. L., Leber, A. B., \& Egeth, H. E. (2008). Top-down control settings and the attentional blink: Evidence for nonspatial contingent capture. Visual Cognition, 5, 616-642. doi: 10.1080/13506280601134018

Folk, C. L, \& Remington, R. W. (1998). Selectivity in distraction by irrelevant featural singletons: evidence for two forms of attentional capture. Journal of Experimental Psychology: Human Perception and Performance, 24, 847-58. doi: 10.1037/00961523.24.3.847 $\overline{\mathrm{wWW}}$

Folk, C. L., Remington, R. W., \& Johnston, J. C. (1992). Involuntary covert orienting is contingent on attentional control settings. Journal of Experimental Psychology: Human Perception and Performance, 18, 1030-1044. doi: 1030-1044.10.1037/00961523.18.4.1030 WwW

Gazzaley, A., \& Nobre, A. C. (2012). Top-down modulation: Bridging selective attention and working memory. Trends in Cognitive Sciences, 16, 129-135. doi: 10.1016/j.tics.2011.11.014 WwW

Ghorashi, S. M., Zuvic, S. M., Visser, T. A. W., \& Di Lollo, V. (2003). Focal distraction: Spatial shifts of attentional focus are not required for contingent capture. Journal of Experimental Psychology: Human Perception and Performance, 29, 78-91. doi: 10.1037/0096-1523.29.1.78 www

Girelli, M., \& Luck, S. J. (1997). Are the same attentional mechanisms used to detect visual search targets defined by color, orientation, and motion? Journal of Cognitive Neuroscience, 9, 238-253. doi: 10.1162/jocn.1997.9.2.238 www

Hackley, S. A. (2009). The speeding of voluntary reaction by a warning signal. Psychophysiology, 46, 225-233. doi: 10.1111/ j.1469-8986.2008.00716.x WwW

Hackley, S. A., \& Valle-Inclán, F. (2003). Which stages of processing are speeded by a warning signal? Biological Psychology, 64, 2745. doi: 10.1016/S0301-0511(03)00101-7 WWW

Hesselmann, G., Niedeggen, M., Sahraie, A., \& Milders, M. (2006). Specifying the distractor inhibition account of attentioninduced motion blindness. Vision Research, 46, 1048-1056. doi: 10.1016/j.visres.2005.10.007 WwW

Horstmann, G., \& Ansorge, U. (2006). Attentional shifts to rare singletons. Visual Cognition, 14, 295-325. doi: $10.1080 / 13506280500236492$ 
Irons, J. L., \& Remington, R. W. (2013). Can attentional control settings be maintained for two color-location conjunctions? Evidence from an RSVP task. Attention Perception \& Psychophysics, 75, 862-875. doi: 10.3758/s13414-013-0439-8 WWW

Kelley, T. A., \& Yantis, S. (2009). Learning to attend: Effects of practice on information selection. Journal of Vision, 9, 1-18. doi: 10.1167/9.7.16 WWW

Lamy, D., Leber, A. B., \& Egeth, H. E. (2004). Effects of task relevance and stimulus-driven salience in feature-search mode. Journal of Experimental Psychology: Human Perception and Performance, 30, 1019-1031. doi: 10.1037/0096-1523.30.6.1019 WWW

Lamy, D., \& Tsal, Y. (1999). A salient distractor does not disrupt conjunction search. Psychonomic Bulletin \& Review, 6, 93-98.

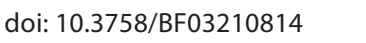

Leblanc, E., \& Jolicœur, P. (2005). The time course of the contingent spatial blink. Canadian Journal of Experimental Psychology, 59, 124-131. doi: 10.1037/h0087467 WWW

Liao, H. I., \& Yeh, S. L. (2013). Capturing attention is not that simple: Different mechanisms for stimulus-driven and contingent capture. Attention, Percepttion, \& Psychophysics, 75, 1703-1714. doi: 10.3758/s13414-013-0537-7 WWW

Maki, W. S., \& Mebane, M. W. (2006). Attentional capture triggers an attentional blink. Psychonomic Bulletin \& Review, 13, 125131. doi: 10.3758/BF03193823

Michael, L., Hesselmann, G., Kiefer, M., \& Niedeggen, M. (2011). Distractor-induced blindness for orientation changes and coherent motion. Vision Research, 51, 1781-1787. doi: 10.1016/j. visres.2011.06.007 WWW

Michael, L., Kiefer, M., \& Niedeggen, M. (2012). The influence of distracter and target features on distracter induced blindness. Advances in Cognitive Psychology, 8, 62-69. doi: 10.2478/ v10053-008-0103-3 wWw

Moore, K. S., \& Weissman, D. H. (2010). Involuntary transfer of a top-down attentional set into the focus of attention: Evidence from a contingent attentional capture paradigm. Attention, Perception, \& Psychophysics, 72, 1495-1509. doi: 10.3758/

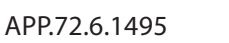

Moore, K. S., \& Weissman, D. H. (2011). Set-specific capture can be reduced by pre-emptively occupying a limited-capacity focus of attention. Visual Cognition, 19, 417-444. doi: 10.1080/13506285.2011.558862 [WW

Müller, H. J., Humphreys, G. W., \& Donnelly, N. (1994). SEarch via Recursive Rejection (SERR): Visual search for single and dual form-conjunction targets. Journal of Experimental Psychology: Human Perception and Performance, 20, 235-258. doi: 10.1037//0096-1523.20.2.235 WWW

Neo, G., \& Chua, F. K. (2006). Capturing focused attention. Perception \& Psychophysics, 68, 1286-1296. doi: 10.3758/ BF03193728 $\overline{\omega W}$
Niedeggen, M., Busch, N. A., \& Winther, G. N. (2015). The state of a central inhibition system predicts access to visual targets: An ERP study on distractor-induced blindness (DIB). Consciousness and Cognition, 35, 308-318. doi: 10.1016/j.concog.2015.02.002 WWW

Niedeggen, M., Hesselmann, G., Sahraie, A., Milders, M., \& Blakemore, C. (2004). Probing the prerequisites for motion blindness. Journal of Cognitive Neuroscience, 16, 584-597. doi: 10.1162/089892904323057317 WW

Niedeggen, M., Michael, L., \& Hesselmann, G. (2012). Closing the gates to consciousness: Distractors activate a central inhibition process. Journal of Cognitive Neuroscience, 24, 1294-1304.

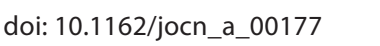

Niedeggen, M., Sahraie, A., Hesselmann, G., Milders, M., \& Blakemore, C. (2002). Is experimental motion blindness due to sensory suppression? An ERP approach. Cognitive Brain Research, 13, 241-247. doi: 10.1016/S0926-6410(01)00122-7 WWW

Niemi, P., \& Näätänen, R. (1981). Foreperiod and simple reaction time. Psychological Bulletin, 89, 133-162. doi: 10.1037/00332909.89.1.133

Olivers, C. N. L., \& Meeter, M. (2008). A boost and bounce theory of temporal attention. Psychological Review, 15, 836-863. doi: 10.1068/v080158 $\overline{\underline{W W}}$

Olivers, C. N. L., \& Watson, D. G. (2006). Input control processes in rapid serial visual presentations: Target selection and distractor inhibition. Journal of Experimental Psychology: Human Perception and Performance, 32, 1083-1092. doi: 10.1037/00961523.32.5.1083

Pashler, H. (1994). Dual-task interference in simple tasks: Data and theory. Psychological Bulletin, 116, 220-244. doi: 10.1037/00332909.116.2.220 $\overline{\mathrm{WWW}}$

Posner, M. I. (1980). Orienting of attention. Quarterly Journal of Experimental Psychology, 32A, 3-25. doi: 10.1080/00335558008248231

Prinzmetal, W., McCool, C., \& Park, S. (2005). Attention: Reaction time and accuracy reveal different mechanisms. Journal of Experimental Psychology, 134, 73-92. doi: 10.1037/0096-3445 134.1.73

Ratcliff, R. (1993). Methods for dealing with reaction time outliers. Psychological Bulletin, 114, 510-532. doi: 10.1037/0033-2909

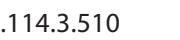

Raymond, J. E., Shapiro, K. L., \& Arnell, K. M. (1992). Temporary suppression of visual processing in an RSVP task: An attentional blink? Journal of Experimental Psychology: Human Perception and Performance, 18, 849-860. doi: 10.1037/0096-

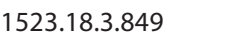

Raymond, J. E., Shapiro, K. L., \& Arnell, K. M. (1995). Similarity determines the attentional blink. Journal of Experimental Psychology: Human Perception and Performance, 21, 653. doi:

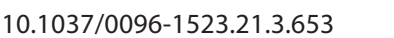


Ruz, M., \& Lupiáñez, J. (2002). A review of attentional capture: On its automaticity and sensitivity to endogenous control. Psicológica, 23, 283-309. URL: http://www.uv.es/revispsi/ articulos2.02/5.RUZ\%26LUPIANEZ.pdf

Sahraie, A., Milders, M., \& Niedeggen, M. (2001). Attention induced motion blindness. Vision Research, 41, 1613-1617. doi: 10.1016/S0042-6989(01)00065-7 WWW

Theeuwes, J., Atchley, P., \& Kramer, A. F. (2000). On the time course of top-down and bottom-up control of visual attention. In S. Monsell \& J. Driver (Eds.), Control of cognitive processes: Attention and performance XVIII (pp. 71-208). Cambridge, MA: The MIT Press.

Treisman, A. (1988). Features and objects: The fourteenth Bartlett memorial lecture. The Quarterly Journal of Experimental Psychology, 40, 201-237. doi: 10.1080/02724988843000104 WWW

van Ede, F., de Lange, F. P., \& Maris, E. (2012). Attentional cues affect accuracy and reaction time via different cognitive and neural processes. The Journal of Neuroscience, 32, 10408-10412. doi: 10.1523/JNEUROSCI.1337-12.2012 WWW

Van Zandt, T. (2002). Analysis of response time distributions. In H. Pashler \& J. Wixted (Eds.), Stevens' handbook of experimental psychology (pp. 461-516). Hoboken, NJ: John Wiley \& Sons. doi: 10.1002/0471214426.pas0412
Velhagen, K., \& Broschmann, D. (2003). Tafeln zur Prüfung des Farbsinns (33rd ed.) [Panels for tests of the color sense]. Stuttgart, Germany: Thieme.

Visser, T. A., Bischof, W., \& Di Lollo, V. (2004). Rapid serial visual distraction: Task-irrelevant items can produce an attentional blink. Perception \& Psychophysics, 66, 1418-1432. doi: 10.3758/ BF03195008 $\overline{\omega W W}$

Wolfe, J. M. (1998). Visual search. In H. Pashler (Ed.), Attention (pp. 13-73). London, UK: Psychology Press.

Woodman, G. F., \& Luck, S. J. (2007). Do the contents of visual working memory automatically influence attentional selection during visual search? Journal of Experimental Psychology: Human Perception and Performance, 33, 363-377. doi: 10.1037/0096-1523.33.2.363 WWW

Zhang, D., Zhou, X., Martens, S. (2009). The impact of negative attentional set upon target processing in RSVP: An ERP study. Neuropsychologia, 47, 2604-2614. doi: 10.3758/s13414-0110207-6. WWW

Zhang, H. H., Zhang, J., \& Kornblum, S. (1999). A parallel distributed processing model of stimulus-stimulus and stimulusresponse compatibility. Cognitive Psychology, 38, 386-432. doi: 10.1006/cogp.1998.0703 $\underline{\underline{W W}}$

Zivony, A., \& Lamy, D. (2014). Attentional engagement is not sufficient to prevent spatial capture. Attention, Perception, \& Psychophysics, 76, 19-31. doi: 10.3758/s13414-013-0543-9 |wWW

RECEIVED 16.03.2016 | ACCEPTED 03.11.2016 